\title{
Facile One-pot Synthesis of Photochromic Pyrans
}

\author{
Weili Zhao and Erick M. Carreira* \\ Laboratorium für Organische Chemie \\ ETH-Hönggerberg HCI H335 \\ CH-8093 Zürich, Switzerland. \\ Carreira@org.chem.ethz.ch
}

\section{Supplementary Material}

General Information: All reactions were performed using oven dried glassware. 1,2dichloroethane was dried over molecular sieves. Naphthols, 2-hydroxydibenzofuran, 1,1diphenyl-2-propyn-1-ol were purchased from either Aldrich or Fluka chemical companies and used without prior purification. 4-hydroxy-1-phenyl-naphthoic acid ethyl ester and propargyl alcohols were prepared by the known procedure. ${ }^{1,2}$ Chromatographic purification of products was accomplished using forced flow chromatography on Fluka Silica Gel 60. NMR spectra were recorded on a Varian Mercury 300 operating at $300 \mathrm{MHz}$ and $75 \mathrm{MHz}$ for ${ }^{1} \mathrm{H}$ and ${ }^{13} \mathrm{C}$, respectively, and referenced to the internal solvent signals. The data is reported as ( $\mathrm{s}=$ singlet, $\mathrm{d}=$ doublet, $\mathrm{t}=$ triplet, $\mathrm{q}=$ quartet, $\mathrm{m}=$ multiplet, coupling constant(s), integration). IR spectra were recorded on a Perkin Elmer Spectrum RX I FT-IR spectrometer and data was reported in unit of $\mathrm{cm}^{-1}$. Mass and high resolution mass spectrometric measurements were performed by the mass spectrometry service of the LOC at the ETHZ. Combustion analysis was performed by the Mikroelementaranalytisches Laboratorium at the ETHZ.

${ }^{1}$ Heller, H.G.; Levell, J. R. U.S. Patent 5955520, 1999.

${ }^{2}$ Moustrou, C.; Rebiere, N.; Samat, A.; Guglielmetti, R.; Yassar, A. E.; Dubest, R.; Aubard, J. Helv. Chim. Acta, 1998, 81, 1293

\section{General Procedure:}

Naphthol or phenol (1 mmol) and propargyl alcohol (1.05-1.1 mmol) in the presence of PPTS $(0.05 \mathrm{mmol})$ and trimethyl orthoformate $(2 \mathrm{mmol})$ in 1,2-dichloroethane $(5 \mathrm{ml})$ were refluxed for 1.5-3.5 hr. Solvent was removed in vacuo, the residue was purified by chromatography and recrystallization.

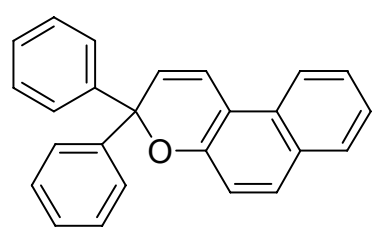

3a. 3,3-diphenyl-[3H]-naphtho-[2,1b]pyran.

IR (KBr): 3060, 1635, 1588, 1490, 1460, 1445, 1241, 1215, 1008, 945, 804, 751, 731, 699. 
${ }^{1} \mathrm{H}-\mathrm{NMR}\left(300 \mathrm{MHz}, \mathrm{CDCl}_{3}\right): \delta 7.96(\mathrm{~d}, \mathrm{~J}=8.4 \mathrm{~Hz}, 1 \mathrm{H}), 7.72(\mathrm{~d}, \mathrm{~J}=8.1 \mathrm{~Hz}, 1 \mathrm{H}), 7.66(\mathrm{~d}, \mathrm{~J}=$ $8.7 \mathrm{~Hz}, 1 \mathrm{H}), 7.52-7.22(\mathrm{~m}, 5 \mathrm{H}), 7.36-7.23(\mathrm{~m}, 8 \mathrm{H}), 7.21(\mathrm{~d}, \mathrm{~J}=9.0 \mathrm{~Hz}, 1 \mathrm{H}), 6.28(\mathrm{~d}, \mathrm{~J}=$ $10.0 \mathrm{~Hz}, 1 \mathrm{H})$.

${ }^{13} \mathrm{C}-\mathrm{NMR}\left(75 \mathrm{MHz}, \mathrm{CDCl}_{3}\right): \delta 150.7,145.0,129.8,129.9,128.6,128.2,127.8,127.6,127.1$, $126.7,123.7,121.4,119.6,118.4,82.5$.

MS: 334.2( $\left.\mathrm{M}^{+}\right)$.

Anal. Calcd for $\mathrm{C}_{25} \mathrm{H}_{18} \mathrm{O}: \mathrm{C}, 89.79 ; \mathrm{H}, 5.43$. Found: C, 89.51; H, 5.63.

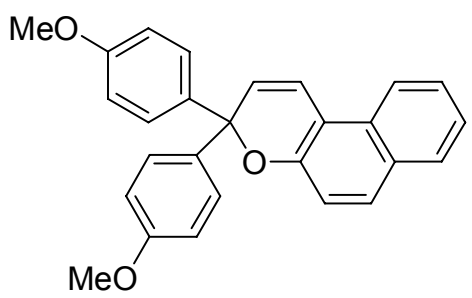

3b. 3,3-di-(p-methoxyphenylphenyl)-[3H]-naphtho[2,1-b]pyran.

IR (KBr): 3055, 2960, 2838, 1628, 1608, 1581 1509, 1460, 1439, 1300, 1249, 1229, 1175, $1081,1030,1000,954,829,814,746,724$.

${ }^{1} \mathrm{H}-\mathrm{NMR}\left(300 \mathrm{MHz}, \mathrm{CDCl}_{3}\right): \delta 7.96(\mathrm{~d}, \mathrm{~J}=8.1 \mathrm{~Hz}, 1 \mathrm{H}), 7.72(\mathrm{~d}, \mathrm{~J}=8.1 \mathrm{~Hz}, 1 \mathrm{H}), 7.66(\mathrm{~d}, \mathrm{~J}=$ $8.7 \mathrm{~Hz}, 1 \mathrm{H}), 7.46(\mathrm{ddd}, \mathrm{J}=8.1,6.8,1.3 \mathrm{~Hz}, 1 \mathrm{H}), 7.42-7.35(\mathrm{~m}, 4 \mathrm{H}), 7.30$ (ddd, J = 8.1, 6.8, $0.9 \mathrm{~Hz}, 1 \mathrm{H}), 7.29(\mathrm{~d}, \mathrm{~J}=10.0 \mathrm{~Hz}, 1 \mathrm{H}), 7.17(\mathrm{~d}, \mathrm{~J}=8.7 \mathrm{~Hz}, 1 \mathrm{H}), 6.88-6.80(\mathrm{~m}, 4 \mathrm{H}), 6.21(\mathrm{~d}, \mathrm{~J}=$ $10.0 \mathrm{~Hz}, 1 \mathrm{H}), 3.77$ (s, 6H).

${ }^{13} \mathrm{C}-\mathrm{NMR}\left(75 \mathrm{MHz}, \mathrm{CDCl}_{3}\right): \delta 158.8,150.5,137.2,129.8,129.7,129.2,128.5,128.3,128.0$, $126.5,123.5,121.3,119.1,118.3,113.9,113.3,82.2,55.2$.

MS: 394.2( $\left.\mathrm{M}^{+}\right)$.

Anal. Calcd for $\mathrm{C}_{27} \mathrm{H}_{22} \mathrm{O}_{3}$ : C, 82.21; H, 5.62. Found: C, 82.10; H, 5.63.

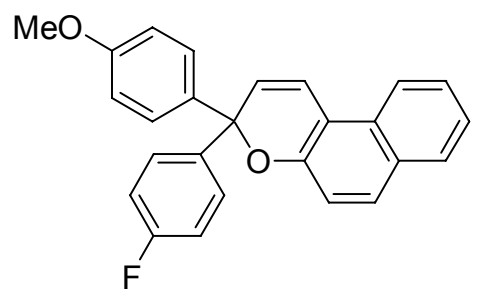

3c. 3-(p-fulorophenyl)-3-(p-methoxyphenyl)-[3H]-naphtho[2,1-b]pyran.

IR (KBr): 3052, 2966, 2932, 2840, 1632, 1600, 1583, 1509, 1458, 1300, 1255, 1230, 1177 , $1157,1080,1000,953,835,817,752$.

${ }^{1} \mathrm{H}-\mathrm{NMR}\left(300 \mathrm{MHz}, \mathrm{CDCl}_{3}\right): \delta 7.96(\mathrm{~d}, \mathrm{~J}=8.1 \mathrm{~Hz}, 1 \mathrm{H}), 7.72(\mathrm{dd}, \mathrm{J}=8.1,0.6 \mathrm{~Hz}, 1 \mathrm{H}), 7.68(\mathrm{~d}$, $\mathrm{J}=8.7 \mathrm{~Hz}, 1 \mathrm{H}), 7.51-7.41(\mathrm{~m}, 3 \mathrm{H}), 7.40-7.36(\mathrm{~m}, 2 \mathrm{H}), 7.33(\mathrm{ddd}, \mathrm{J}=7.8,6.8,0.9 \mathrm{~Hz}, 1 \mathrm{H})$, $7.31(\mathrm{~d}, \mathrm{~J}=10.0 \mathrm{~Hz}, 1 \mathrm{H}), 7.17(\mathrm{~d}, \mathrm{~J}=8.7 \mathrm{~Hz}, 1 \mathrm{H}), 7.05-6.94(\mathrm{~m}, 2 \mathrm{H}), 6.89-6.80(\mathrm{~m}, 2 \mathrm{H}), 6.20$ $(\mathrm{d}, \mathrm{J}=10.0 \mathrm{~Hz}, 1 \mathrm{H}), 3.77(\mathrm{~s}, 3 \mathrm{H})$.

${ }^{13} \mathrm{C}-\mathrm{NMR}\left(75 \mathrm{MHz}, \mathrm{CDCl}_{3}\right): \delta 162.0\left({ }^{1} \mathrm{~J}=246.6 \mathrm{~Hz}\right), 159.0,150.3,140.9,136.7,129.9,129.7$, $129.3,128.7\left({ }^{3} \mathrm{~J}=7.3 \mathrm{~Hz}\right), 128.5,128.3,127.6,126.6,123.6,121.3,119.5,118.2,114.8\left({ }^{2} \mathrm{~J}=\right.$ 22.0Hz), 113.4, 81.9, 55.2.

MS: 382.2( $\left.\mathrm{M}^{+}\right)$.

Anal. Calcd for $\mathrm{C}_{26} \mathrm{H}_{19} \mathrm{FO}_{2}$ : C, 81.66; H, 5.01; F, 4.97. Found: C, 81.55; H, 5.13; F, 5.06.

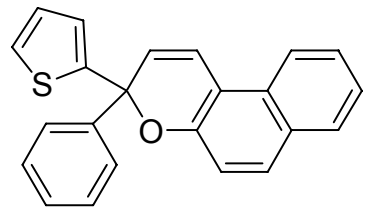


3d. 3-phenyl-3-(thiophene-2-yl)-[3H]-naphtho[2,1-b]pyran.

IR (KBr): 3064, 1632, 1492, 1446, 1229, 1205, 1006, 813, 752, 709, 693.

${ }^{1} \mathrm{H}-\mathrm{NMR}\left(300 \mathrm{MHz}, \mathrm{CDCl}_{3}\right): \delta 7.97(\mathrm{~d}, \mathrm{~J}=8.4 \mathrm{~Hz}, 1 \mathrm{H}), 7.73(\mathrm{~d}, \mathrm{~J}=8.1 \mathrm{~Hz}, 1 \mathrm{H}), 7.68(\mathrm{~d}, \mathrm{~J}=$ $9.0 \mathrm{~Hz}, 1 \mathrm{H}), 7.62-7.54(\mathrm{~m}, 2 \mathrm{H}), 7.48(\mathrm{ddd}, \mathrm{J}=8.3,6.8,1.3 \mathrm{~Hz}, 1 \mathrm{H}), 7.40-7.27(\mathrm{~m}, 5 \mathrm{H}), 7.22$ $(\mathrm{d}, \mathrm{J}=8.7 \mathrm{~Hz}, 1 \mathrm{H}), 6.98-6.88(\mathrm{~m}, 2 \mathrm{H}), 6.30(\mathrm{~d}, \mathrm{~J}=10.0 \mathrm{~Hz}, 1 \mathrm{H})$.

${ }^{13} \mathrm{C}-\mathrm{NMR}\left(75 \mathrm{MHz}, \mathrm{CDCl}_{3}\right): \delta 150.3,149.3,144.4,130.0,129.7,129.4,128.5,128.1,127.8$, 127.2, 126.7, 126.4, 126.3, 123.7, 121.3, 119.7, 118.3, 113.8, 80.3.

MS: 340.1 $\left(\mathrm{M}^{+}\right)$.

Anal. Calcd for $\mathrm{C}_{23} \mathrm{H}_{16} \mathrm{OS}$ : C, 81.14; H, 4.74; S, 9.42. Found: C, 81.14; H, 4.94; S, 9.39.

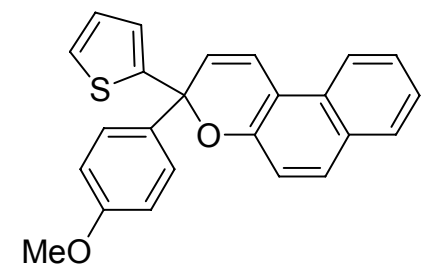

3e. 3-(p-methoxyphenyl)-3-(thiophene-2-yl)-[3H]-naphtho[2,1-b]pyran.

IR (KBr): 3061, 3009, 2962, 2833, 1628, 1609, 1584 1510, 1459, 1436, 1304, 1250, 1234, $1174,1033,1000,825,804,753,700$.

${ }^{1} \mathrm{H}-\mathrm{NMR}\left(300 \mathrm{MHz}, \mathrm{CDCl}_{3}\right): \delta 7.97(\mathrm{~d}, \mathrm{~J}=8.4 \mathrm{~Hz}, 1 \mathrm{H}), 7.73(\mathrm{~d}, \mathrm{~J}=8.4 \mathrm{~Hz}, 1 \mathrm{H}), 7.67(\mathrm{~d}, \mathrm{~J}=$ $8.7 \mathrm{~Hz}, 1 \mathrm{H}), 7.54-7.42(\mathrm{~m}, 3 \mathrm{H}), 7.37-7.26(\mathrm{~m}, 3 \mathrm{H}), 7.19(\mathrm{~d}, \mathrm{~J}=8.7 \mathrm{~Hz}, 1 \mathrm{H}), 6.98-6.90(\mathrm{~m}, 2 \mathrm{H})$, 6.90-6.80 (m, 2H), $6.26(\mathrm{~d}, \mathrm{~J}=10.0 \mathrm{~Hz}, 1 \mathrm{H}), 3.78$ (s, 3H).

${ }^{13} \mathrm{C}-\mathrm{NMR}\left(75 \mathrm{MHz}, \mathrm{CDCl}_{3}\right.$ ): $\delta 159.3,150.4,149.8,136.7,130.0,129.9,129.5,128.6,127.9$, 127.5, 126.7, 126.4, 126.3, 126.2, 123.8, 121.4, 119.6, 118.4, 113.9, 113.5, 80.2, 55.2.

MS: $370.2\left(\mathrm{M}^{+}\right)$

Anal. Calcd for $\mathrm{C}_{24} \mathrm{H}_{18} \mathrm{O}_{2} \mathrm{~S}$ : C, 77.81; H, 4.90; S, 8.66. Found: C, 77.74; H, 4.95; S, 8.62.

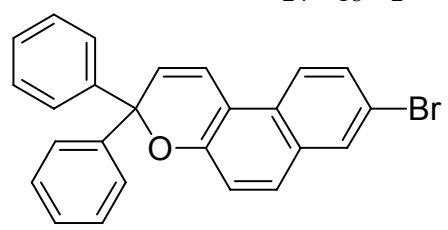

4a. 8-bromo-3,3-diphenyl-[3H]-naphtho-[2,1b]pyran.

IR (KBr): 3056, 1628, 1580, 1491, 1446, 1210, 1184, 1007, 960, 877, 806, 767, 742, 728, $706,694$.

${ }^{1} \mathrm{H}-\mathrm{NMR}\left(300 \mathrm{MHz}, \mathrm{CDCl}_{3}\right): \delta 7.86(\mathrm{~d}, \mathrm{~J}=1.9 \mathrm{~Hz}, 1 \mathrm{H}), 7.81(\mathrm{~d}, \mathrm{~J}=9.0 \mathrm{~Hz}, 1 \mathrm{H}), 7.56(\mathrm{~d}, \mathrm{~J}=$ 9.0Hz, 1H), 7.56-7.40 (m, 5H), 7.38-7.14 (m, 8H), $6.29(\mathrm{~d}, \mathrm{~J}=10.0 \mathrm{~Hz}, 1 \mathrm{H})$.

${ }^{13} \mathrm{C}-\mathrm{NMR}\left(75 \mathrm{MHz}, \mathrm{CDCl}_{3}\right): \delta 150.9,144.7,130.6,130.5,129.9,128.9,128.3,128.2,127.7$, 127.1, 123.2, 119.5, 119.1, 117.3, 114.2, 82.7.

MS: $412.1\left(\mathrm{M}^{+}\right)$.

Anal. Calcd for $\mathrm{C}_{25} \mathrm{H}_{17} \mathrm{OBr}$ : C, 72.65; H, 4.15; Br, 19.33. Found: C, 72.65; H, 4.32; Br, 19.43.

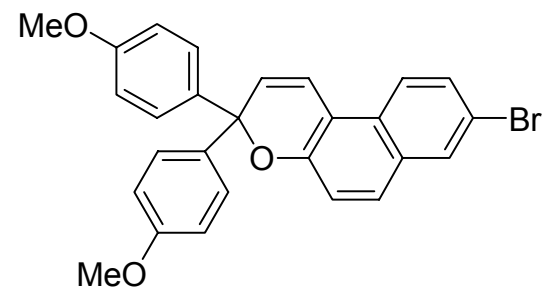

4b. 8-bromo-3,3-di-(p-methoxyphenyl)-[3H]-naphtho[2,1-b]pyran.

IR (KBr): 3055, 3010, 2951, 2832, 1628, 1607, 1579 1507, 1462, 1439, 1300, 1249, 1176, $1033,1002,954,833,814,753,700$. 
${ }^{1} \mathrm{H}-\mathrm{NMR}\left(300 \mathrm{MHz}, \mathrm{CDCl}_{3}\right): \delta 7.85(\mathrm{~d}, \mathrm{~J}=1.9 \mathrm{~Hz}, 1 \mathrm{H}), 7.81(\mathrm{~d}, \mathrm{~J}=8.7 \mathrm{~Hz}, 1 \mathrm{H}), 7.54(\mathrm{~d}, \mathrm{~J}=$ $9.0 \mathrm{~Hz}, 1 \mathrm{H}), 7.51(\mathrm{dd}, \mathrm{J}=9.0,1.9 \mathrm{~Hz}, 1 \mathrm{H}), 7.42-7.33(\mathrm{~m}, 4 \mathrm{H}), 7.21(\mathrm{~d}, \mathrm{~J}=10.2 \mathrm{~Hz}, 1 \mathrm{H}), 7.17$ $(\mathrm{d}, \mathrm{J}=9.0 \mathrm{~Hz}, 1 \mathrm{H}), 6.88-6.80(\mathrm{~m}, 4 \mathrm{H}), 6.22(\mathrm{~d}, 1 \mathrm{H}, \mathrm{J}=9.6 \mathrm{~Hz}), 3.77(\mathrm{~s}, 6 \mathrm{H})$.

${ }^{13} \mathrm{C}-\mathrm{NMR}\left(75 \mathrm{MHz}, \mathrm{CDCl}_{3}\right): \delta 150.9,137.1,130.5,129.8,128.8,128.7,128.4,123.3,119.5$, $118.8,117.3,114.1,113.5,82.4,55.2$.

Anal. Calcd for $\mathrm{C}_{27} \mathrm{H}_{21} \mathrm{BrO}_{3}$ : C, 68.51; H, 4.47; Br, 16.88. Found: C, 68.31; H, 4.66; $\mathrm{Br}$, 16.86.

HRMS (MALDI) Calcd. for $\mathrm{C}_{27} \mathrm{H}_{21} \mathrm{BrO}_{3}: 473.0747\left(\mathrm{MH}^{+}\right)$. Found: 473.0752 (93\%).

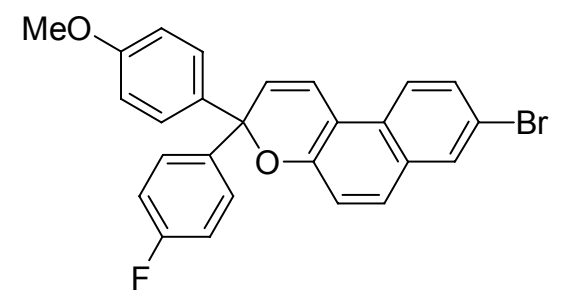

4c.8-bromo-3-(p-fulorophenyl)-3-p-methoxyphenyl-[3H]-naphtho[2,1-b]pyran.

IR (KBr): 3051, 2928, 2835, 1627, 1600, 1581, 1508, 1464, 1300, 1249, 1218, 1176, 1159 , $1004,961,837,727$.

${ }^{1} \mathrm{H}-\mathrm{NMR}\left(300 \mathrm{MHz}, \mathrm{CDCl}_{3}\right): \delta 7.85(\mathrm{~d}, \mathrm{~J}=1.9 \mathrm{~Hz}, 1 \mathrm{H}), 7.81(\mathrm{~d}, \mathrm{~J}=8.7 \mathrm{~Hz}, 1 \mathrm{H}), 7.54(\mathrm{~d}, \mathrm{~J}=$ $9.0 \mathrm{~Hz}, 1 \mathrm{H}), 7.51(\mathrm{dd}, \mathrm{J}=9.0,2.2 \mathrm{~Hz}, 1 \mathrm{H}), 7.46-7.39(\mathrm{~m}, 2 \mathrm{H}), 7.38-7.31(\mathrm{~m}, 2 \mathrm{H}), 7.24(\mathrm{~d}, \mathrm{~J}=$ $10.2 \mathrm{~Hz}, 1 \mathrm{H}), 7.17(\mathrm{~d}, \mathrm{~J}=9.0 \mathrm{~Hz}, 1 \mathrm{H}), 7.04-6.95(\mathrm{~m}, 2 \mathrm{H}), 6.88-6.80(\mathrm{~m}, 2 \mathrm{H}), 6.20(\mathrm{~d}, \mathrm{~J}=$ $10.0 \mathrm{~Hz}, 1 \mathrm{H}), 3.77$ (s, 3H).

${ }^{13} \mathrm{C}-\mathrm{NMR}\left(75 \mathrm{MHz}, \mathrm{CDCl}_{3}\right): \delta 162.2\left({ }^{1} \mathrm{~J}=246.6 \mathrm{~Hz}\right), 159.2,150.7,140.7,136.6,130.6,130.5$, $129.9,129.0,128.9\left({ }^{3} \mathrm{~J}=7.3 \mathrm{~Hz}\right), 128.4,128.3,128.3,123.2,119.4,119.1,117.4,115.0\left({ }^{2} \mathrm{~J}=\right.$ $22.0 \mathrm{~Hz}), 114.2,113.6,82.1,55.2$.

MS: $460.0\left(\mathrm{M}^{+}\right)$.

Anal. Calcd for $\mathrm{C}_{27} \mathrm{H}_{21} \mathrm{BrFO}_{2}$ : C, 67.69; H, 3.93; Br, 17.32; F, 4.12. Found: C, 67.48; H, 4.08; $\mathrm{Br}, 17.23 ; \mathrm{F}, 4.15$.

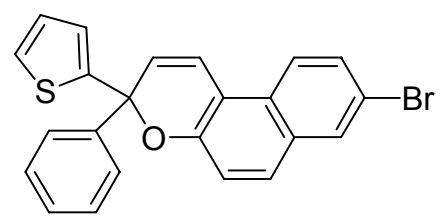

4d.8-bromo-3-phenyl-3-(thiophene-2-yl)-[3H]-naphtho[2,1-b]pyran.

IR (KBr): 3051, 3020, 1628, 1583, 1497, 1446, 1228, 1213, 1170, 1093, 1066, 996, 948, 876, 809, 703, 692.

${ }^{1} \mathrm{H}-\mathrm{NMR}\left(300 \mathrm{MHz}, \mathrm{CDCl}_{3}\right): \delta 7.87(\mathrm{~d}, \mathrm{~J}=1.9 \mathrm{~Hz}, 1 \mathrm{H}), 7.82(\mathrm{~d}, \mathrm{~J}=9.0 \mathrm{~Hz}, 1 \mathrm{H}), 7.64-7.48(\mathrm{~m}$, $4 \mathrm{H}), 7.40-7.22(\mathrm{~m}, 5 \mathrm{H}), 7.22(\mathrm{~d}, \mathrm{~J}=8.7 \mathrm{~Hz}, 1 \mathrm{H}), 6.98-6.87(\mathrm{~m}, 2 \mathrm{H}), 6.30(\mathrm{~d}, \mathrm{~J}=9.7 \mathrm{~Hz}, 1 \mathrm{H})$.

${ }^{13} \mathrm{C}-\mathrm{NMR}\left(75 \mathrm{MHz}, \mathrm{CDCl}_{3}\right): \delta 150.6,149.2,144.3,130.7,130.5,130.0,129.1,128.4,128.3$, $128.0,127.9,126.6,126.5,126.5,126.4,123.2,119.5,119.4,117.5,114.0,80.5$.

MS: $418.0\left(\mathrm{M}^{+}\right)$.

Anal. Calcd for $\mathrm{C}_{23} \mathrm{H}_{15}$ BrOS: C, 65.88; H, 3.61; Br, 19.05; S, 7.65. Found: C, 65.65; H, 3.80; $\mathrm{Br}, 18.93 ; \mathrm{S}, 7.61$.

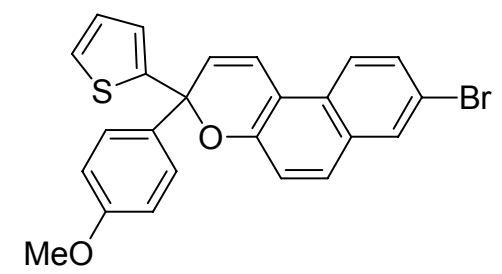


4e.8-bromo-3-(p-methoxyphenyl)-3-(thiophene-2-yl)-[3H]-naphtho[2,1-b]pyran.

IR (KBr): 3066, 2950, 2837, 1636, 1607, 1580, 1508, 1460, 1304, 1255, 1215, 1164, 1067, $1030,995,942,892,814,703$.

${ }^{1} \mathrm{H}-\mathrm{NMR}\left(300 \mathrm{MHz}, \mathrm{CDCl}_{3}\right): \delta 7.87(\mathrm{~d}, \mathrm{~J}=1.9 \mathrm{~Hz}, 1 \mathrm{H}), 7.82(\mathrm{~d}, \mathrm{~J}=9.0 \mathrm{~Hz}, 1 \mathrm{H}), 7.57(\mathrm{~d}, \mathrm{~J}=$ $9.0 \mathrm{~Hz}, 1 \mathrm{H}), 7.52(\mathrm{dd}, \mathrm{J}=9.0,2.2 \mathrm{~Hz}, 1 \mathrm{H}), 7.50-7.44(\mathrm{~m}, 2 \mathrm{H}), 7.31-7.27(\mathrm{~m}, 1 \mathrm{H}), 7.23(\mathrm{~d}, \mathrm{~J}=$ $9.0 \mathrm{~Hz}, 1 \mathrm{H}), 7.20(\mathrm{~d}, \mathrm{~J}=8.4 \mathrm{~Hz}, 1 \mathrm{H}), 6.96-6.82(\mathrm{~m}, 4 \mathrm{H}), 6.27(\mathrm{~d}, \mathrm{~J}=10.0 \mathrm{~Hz}, 1 \mathrm{H}), 3.78(\mathrm{~s}, 3 \mathrm{H})$.

${ }^{13} \mathrm{C}-\mathrm{NMR}\left(75 \mathrm{MHz}, \mathrm{CDCl}_{3}\right): \delta 159.4,150.6,149.5,136.4,130.7,130.5,129.9,129.0,128.3$, $128.0,127.8,126.5,126.3,123.2,119.5,119.1,117.4,114.0,113.5,80.3,55.2$.

MS: $447.1\left(\mathrm{M}^{+}\right)$.

Anal. Calcd for $\mathrm{C}_{24} \mathrm{H}_{17} \mathrm{BrO}_{2} \mathrm{~S}: \mathrm{C}, 64.15 ; \mathrm{H}, 3.81 ; \mathrm{Br}, 17.78 ; \mathrm{S}, 7.14$. Found: $\mathrm{C}, 64.11 ; \mathrm{H}, 3.95$; $\mathrm{Br}, 17.81 ; \mathrm{S}, 7.12$.

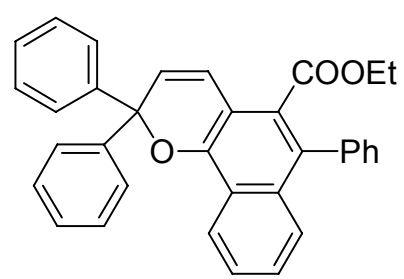

5a. 2,2,6-triphenyl-5-ethoxycarbonyl-[2H]naphtho[1,2-b]pyran.

IR (KBr): 3056, 2980, 2920, 2865, 1716, 1642, 1490, 1448, 1398, 1366, 1230, 1165, 1041, $1000,938,763,700$.

${ }^{1} \mathrm{H}-\mathrm{NMR}\left(200 \mathrm{MHz}, \mathrm{CDCl}_{3}\right): \delta 8.47-8.38(\mathrm{~m}, 1 \mathrm{H}), 7.60-7.46(\mathrm{~m}, 6 \mathrm{H}), 7.46-7.20(\mathrm{~m}, 10 \mathrm{H})$, $6.83(\mathrm{~d}, \mathrm{~J}=10.0 \mathrm{~Hz}, 1 \mathrm{H}), 6.24(\mathrm{~d}, \mathrm{~J}=10.0 \mathrm{~Hz}, 1 \mathrm{H}), 3.98(\mathrm{q}, \mathrm{J}=7.1 \mathrm{~Hz}, 2 \mathrm{H}), 0.87(\mathrm{t}, \mathrm{J}=7.1 \mathrm{~Hz}$, $3 \mathrm{H})$.

${ }^{13} \mathrm{C}-\mathrm{NMR}\left(75 \mathrm{MHz}, \mathrm{CDCl}_{3}\right): \delta 168.6,147.6,145.0,138.3,133.0,131.0,130.6,128.9,128.3$, 128.1, 128.0, 127.7, 127.4, 127.0, 126.6, 125.1, 122.1, 121.1, 112.1, 83.2, 61.0, 13.4.

HRMS (MALDI) Calcd for $\mathrm{C}_{34} \mathrm{H}_{26} \mathrm{O}_{3}: 505.1774\left(\mathrm{MNa}^{+}\right)$. Found: $505.1776(67 \%)$.

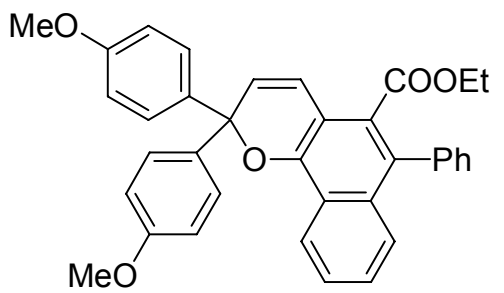

5b. 2,2-di-(p-methoxyphenyl)-5-ethoxycarbonyl-6-phenyl[2H]naphtho[1,2-b]pyran.

IR (KBr): 3062, 2933, 2902, 2835, 1721, 1607, 1509, 1463, 1444, 1373, 1292, 1252, 1230, $1173,1038$.

${ }^{1} \mathrm{H}-\mathrm{NMR}\left(300 \mathrm{MHz}, \mathrm{CDCl}_{3}\right): \delta 8.41-8.36(\mathrm{~m}, 1 \mathrm{H}), 7.56-7.28(\mathrm{~m}, 12 \mathrm{H}), 6.90-6.83(\mathrm{~m}, 4 \mathrm{H})$, $6.79(\mathrm{~d}, \mathrm{~J}=10.0 \mathrm{~Hz}, 1 \mathrm{H}), 6.17(\mathrm{~d}, \mathrm{~J}=10.0 \mathrm{~Hz}, 1 \mathrm{H}), 3.98(\mathrm{q}, \mathrm{J}=7.1 \mathrm{~Hz}, 2 \mathrm{H}), 3.79(\mathrm{~s}, 6 \mathrm{H}), 0.87$ (t, J $=7.1 \mathrm{~Hz}, 3 \mathrm{H})$.

${ }^{13} \mathrm{C}-\mathrm{NMR}\left(75 \mathrm{MHz}, \mathrm{CDCl}_{3}\right): \delta 168.5,159.0,147.5,138.3,137.3,132.8,130.7,130.6,128.8$, $128.4,128.3,128.0,127.4,126.9,126.4,125.1,122.1,120.6,113.5,112.1,110.0,82.8,61.0$, 55.3, 13.6.

MS: $542.1\left(\mathrm{M}^{+}\right)$.

Anal. Calcd for $\mathrm{C}_{36} \mathrm{H}_{30} \mathrm{O}_{5}$ : C, 79.69; H, 5.57. Found: C, 79.47; H, 5.84. 


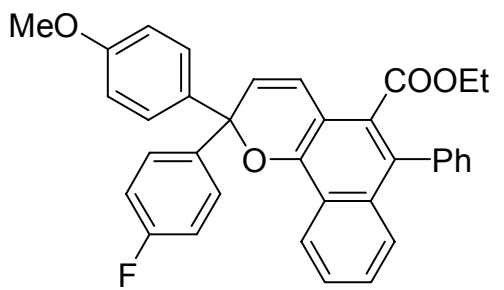

5c. 5-ethoxycarbonyl-2-(p-fluorophenyl)-2-( $p$-methoxyphenyl)-6-phenyl[2H]naphtho[1,2b]pyran.

IR (KBr): 3057, 2979, 2835, 1719, 1605, 1506, 1444, 1372, 1291, 1253, 1227, 1175, 1158 , $1040,997$.

${ }^{1} \mathrm{H}-\mathrm{NMR}\left(200 \mathrm{MHz}, \mathrm{CDCl}_{3}\right): \delta 8.43-8.35(\mathrm{~m}, 1 \mathrm{H}), 7.60-7.28(\mathrm{~m}, 12 \mathrm{H}), 7.10-6.96(\mathrm{~m}, 2 \mathrm{H})$, $6.92-6.83(\mathrm{~m}, 2 \mathrm{H}), 6.83(\mathrm{~d}, \mathrm{~J}=10.0 \mathrm{~Hz}, 1 \mathrm{H}), 6.17(\mathrm{~d}, \mathrm{~J}=10.0 \mathrm{~Hz}, 1 \mathrm{H}), 4.00(\mathrm{q}, \mathrm{J}=7.1 \mathrm{~Hz}, 2 \mathrm{H})$, $3.79(\mathrm{~s}, 3 \mathrm{H}), 0.88(\mathrm{t}, \mathrm{J}=7.1 \mathrm{~Hz}, 3 \mathrm{H})$.

${ }^{13} \mathrm{C}-\mathrm{NMR}\left(75 \mathrm{MHz}, \mathrm{CDCl}_{3}\right): \delta 168.6,162.2\left({ }^{1} \mathrm{~J}=247.8 \mathrm{~Hz}\right), 147.4,141.1,138.3,136.9,133.0$, $131.1,130.6,128.8\left({ }^{3} \mathrm{~J}=7.3 \mathrm{~Hz}\right), 128.4,128.0,127.5,127.1,127.0,126.7,125.1,122.0$, $121.1,115.1\left({ }^{2} \mathrm{~J}=22.0 \mathrm{~Hz}\right), 113.7,112.1,111.0,82.6,61.0,55.2,13.4$.

HRMS (MALDI) Calcd for $\mathrm{C}_{35} \mathrm{H}_{27} \mathrm{FO}_{4}: 553.1786\left(\mathrm{MNa}^{+}\right)$. Found: 553.1784 (12\%).

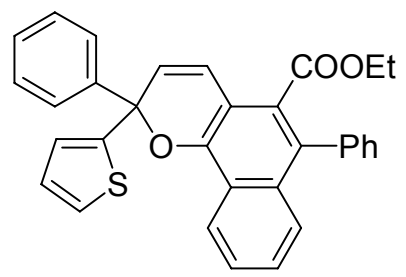

5d. 2,6-diphenyl-5-ethoxycarbonyl-2-(thiophene-2-yl)-[2H]naphtho[1,2-b]pyran.

IR (KBr): 3056, 2977, 2933, 1717, 1650, 1599, 1503, 1444, 1393, 1372, 1293, 1258, 1230, $1169,1145,1114,1041,990$.

${ }^{1} \mathrm{H}-\mathrm{NMR}\left(300 \mathrm{MHz}, \mathrm{CDCl}_{3}\right): \delta 8.47-8.38(\mathrm{~m}, 1 \mathrm{H}), 7.65-7.56(\mathrm{~m}, 2 \mathrm{H}), 7.55-7.47(\mathrm{~m}, 2 \mathrm{H})$, 7.46-7.27 (m, 10H), $7.04(\mathrm{dd}, \mathrm{J}=3.4,1.2 \mathrm{~Hz}, 1 \mathrm{H}), 6.94(\mathrm{dd}, \mathrm{J}=5.0,3.4 \mathrm{~Hz}, 1 \mathrm{H}), 6.82(\mathrm{~d}, \mathrm{~J}=$ $9.7 \mathrm{~Hz}, 1 \mathrm{H}), 6.25(\mathrm{~d}, \mathrm{~J}=9.7 \mathrm{~Hz}, 1 \mathrm{H}), 3.98(\mathrm{q}, 2 \mathrm{H}, \mathrm{J}=7.1 \mathrm{~Hz}), 0.87(\mathrm{t}, 3 \mathrm{H}, \mathrm{J}=7.1 \mathrm{~Hz})$.

${ }^{13} \mathrm{C}-\mathrm{NMR}\left(75 \mathrm{MHz}, \mathrm{CDCl}_{3}\right): \delta 168.5,149.5,147.4,144.6,138.3,133.0,131.3,130.6,128.8$, $128.3,128.0,127.7,127.5,127.2,127.0,126.7,126.5,126.4,126.3,125.2,122.3,121.3$, 112.0, 80.9, 61.0, 13.4 .

HRMS (MALDI) Calcd for $\mathrm{C}_{32} \mathrm{H}_{24} \mathrm{O}_{3} \mathrm{~S}: 511.1338\left(\mathrm{MNa}^{+}\right.$). Found: 511.1335 (15\%).

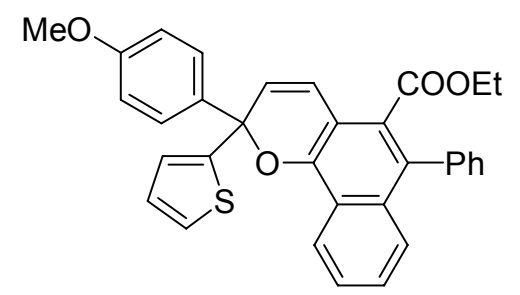

5e. 5-ethoxycarbonyl-2-(p-methoxyphenyl)-2-(thiophene-2-yl)-6-phenyl[2H]naphtho[1,2b]pyran.

IR (KBr): 3056, 2985, 2950, 2832, 1727, 1606,1508, 1442, 1368, 1249, 1224, 1164, 1038, 946, 816, 770, 702 .

${ }^{1} \mathrm{H}-\mathrm{NMR}\left(300 \mathrm{MHz}, \mathrm{CDCl}_{3}\right): \delta 8.44-8.38(\mathrm{~m}, 1 \mathrm{H}), 7.56-7.47(\mathrm{~m}, 4 \mathrm{H}), 7.46-7.36(\mathrm{~m}, 4 \mathrm{H})$, 7.35-7.28 (m, 3H), $7.01(\mathrm{dd}, \mathrm{J}=3.4,1.2 \mathrm{~Hz}, 1 \mathrm{H}), 6.95(\mathrm{dd}, \mathrm{J}=5.0,3.5 \mathrm{~Hz}, 1 \mathrm{H}), 6.92-6.85(\mathrm{~m}$, $2 \mathrm{H}), 6.80(\mathrm{~d}, \mathrm{~J}=10.0 \mathrm{~Hz}, 1 \mathrm{H}), 6.22(\mathrm{~d}, 1 \mathrm{H}, \mathrm{J}=10.0 \mathrm{~Hz}), 3.98(\mathrm{q}, \mathrm{J}=7.1 \mathrm{~Hz}, 2 \mathrm{H}), 3.80(\mathrm{~s}, 3 \mathrm{H})$, $0.87(\mathrm{t}, \mathrm{J}=7.1 \mathrm{~Hz}, 3 \mathrm{H})$. 
${ }^{13} \mathrm{C}-\mathrm{NMR}\left(75 \mathrm{MHz}, \mathrm{CDCl}_{3}\right): \delta 168.6,159.4,149.8,147.3,138.3,136.7,133.0,131.2,130.6$, $128.8,128.0,127.8,127.8,127.5,127.1,127.0,126.6,126.5,126.4,126.1,125.2,122.3$, 121.1, 113.6, 112.0, 80.8, 61.0, 55.2, 13.4 .

MS: $518.1\left(\mathrm{M}^{+}\right)$.

Anal. Calcd for $\mathrm{C}_{33} \mathrm{H}_{26} \mathrm{O}_{4} \mathrm{~S}$ : C, 76.42; H, 5.05; S, 6.18. Found: C, 76.22; H, 5.09; S, 6.12.

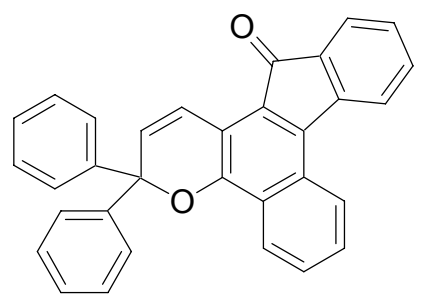

6a. 3,3-diphenyl-13-oxo-3H, 13H-indeno[2,1-f]naphtho[1,2-b] pyran.

IR (KBr): 3055, 1701, 1601, 1558, 1490, 1463, 1450, 1398, 1369, 1338, 1274, 1195, 1133, $1112,1076,955,770,755,699,674$.

${ }^{1} \mathrm{H}$ NMR $\left(300 \mathrm{MHz}, \mathrm{CDCl}_{3}\right): \delta 8.43-8.36(\mathrm{~m}, 1 \mathrm{H}), 8.36-8.28(\mathrm{~m}, 1 \mathrm{H}), 7.91(\mathrm{~d}, \mathrm{~J}=10.0 \mathrm{~Hz}$, $1 \mathrm{H}), 7.82(\mathrm{~d}, \mathrm{~J}=7.8 \mathrm{~Hz}, 1 \mathrm{H}), 7.60-7.48(\mathrm{~m}, 7 \mathrm{H}), 7.41(\mathrm{td}, \mathrm{J}=7.4,1.3 \mathrm{~Hz}, 1 \mathrm{H}), 7.37-7.22(\mathrm{~m}$, $6 \mathrm{H}), 7.18(\mathrm{t}, \mathrm{J}=7.4 \mathrm{~Hz}, 1 \mathrm{H}), 6.29(\mathrm{~d}, \mathrm{~J}=10.0 \mathrm{~Hz}, 1 \mathrm{H})$.

${ }^{13} \mathrm{C}$ NMR $\left(75 \mathrm{MHz}, \mathrm{CDCl}_{3}\right): \delta 195.6,149.0,144.7,144.4,135.4,134.4,134.2,129.8,129.2$, $128.1,127.8,127.7,127.6,127.4,126.8,126.1,124.6,123.5,123.4,122.2,119.6,113.3$, 108.8, 83.3.

HRMS (MALDI) Calcd. for $\mathrm{C}_{32} \mathrm{H}_{20} \mathrm{O}_{2}: 437.1536\left(\mathrm{MH}^{+}\right)$. Found: 437.1532 (100\%).

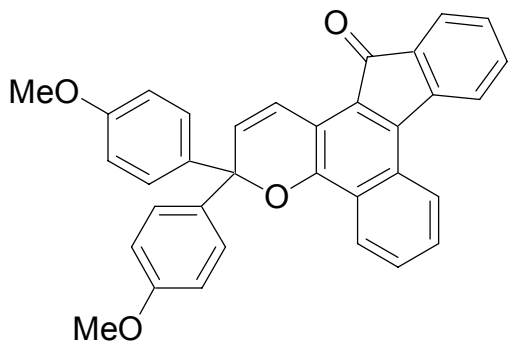

6b. 3,3-di( $p$-methoxyphenyl)-13-oxo-3H, 13H-indeno[2,1-f]naphtho[1,2-b] pyran.

IR (KBr): 3063, 2932, 2836, 1705, 1605, 1583, 1507, 1463, 1401, 1368, 1278, 1246, 1175, $1079,1031,831,778,716,664$.

${ }^{1} \mathrm{H}$ NMR $\left(300 \mathrm{MHz}, \mathrm{CDCl}_{3}\right): \delta 8.40-8.30(\mathrm{~m}, 2 \mathrm{H}), 7.88(\mathrm{~d}, \mathrm{~J}=10.0 \mathrm{~Hz}, 1 \mathrm{H}), 7.86(\mathrm{~d}, \mathrm{~J}=$ $7.4 \mathrm{~Hz}, 1 \mathrm{H}), 7.58(\mathrm{~d}, \mathrm{~J}=7.8 \mathrm{~Hz}, 1 \mathrm{H}), 7.57-7.48(\mathrm{~m}, 2 \mathrm{H}), 7.47-7.34(\mathrm{~m}, 5 \mathrm{H}), 7.19(\mathrm{t}, \mathrm{J}=7.4 \mathrm{~Hz}$, $1 \mathrm{H}), 7.00-6.78(\mathrm{~m}, 4 \mathrm{H}), 6.29(\mathrm{~d}, \mathrm{~J}=10.0 \mathrm{~Hz}, 1 \mathrm{H}), 3.77(\mathrm{~s}, 6 \mathrm{H})$.

${ }^{13} \mathrm{C}$ NMR $\left(75 \mathrm{MHz}, \mathrm{CDCl}_{3}\right): \delta 196.1,159.2,149.4,145.1,137.1,135.4,134.7,134.4,130.4$, 129.4, 128.4, 128.0, 127.8, 127.6, 124.8, 123.7, 122.4, 119.4, 113.6, 113.5, 83.3, 55.2.

HRMS (MALDI) Calcd. for $\mathrm{C}_{34} \mathrm{H}_{24} \mathrm{O}_{4}: 497.1747\left(\mathrm{MH}^{+}\right)$. Found: $497.1742(100 \%)$.

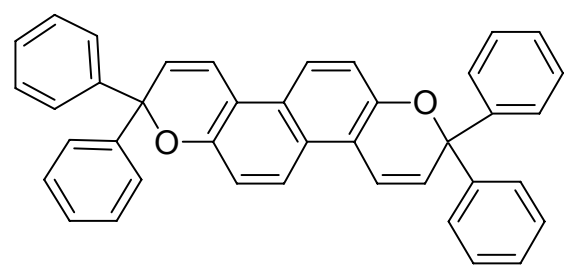

7a. 3,3,9,9-tetraphenyl-3H,9H-naphtho[2,1-b:6,5b']dipyran

IR (KBr): 3058, 1626, 1520, 1490, 1447, 1380, 1253, 1068, 749, 700. 
${ }^{1} \mathrm{H}-\mathrm{NMR}\left(300 \mathrm{MHz}, \mathrm{CDCl}_{3}\right): \delta 7.79(\mathrm{~d}, \mathrm{~J}=9.0 \mathrm{~Hz}, 2 \mathrm{H}), 7.52-7.44(\mathrm{~m}, 8 \mathrm{H}), 7.36-7.14(\mathrm{~m}$, $16 \mathrm{H}), 6.27(\mathrm{~d}, \mathrm{~J}=10.0 \mathrm{~Hz}, 2 \mathrm{H})$.

${ }^{13} \mathrm{C}-\mathrm{NMR}\left(75 \mathrm{MHz}, \mathrm{CDCl}_{3}\right): \delta 148.9,144.7,128.3,128.0,127.5,126.9,125.5,123.0,119.6$, $118.8,114.7,82.2$.

MS: 540.3.

Anal. Calcd for $\mathrm{C}_{40} \mathrm{H}_{28} \mathrm{O}_{2}$ : C, 88.86; H, 5.22. Found: C, 88.68; H, 5.42.

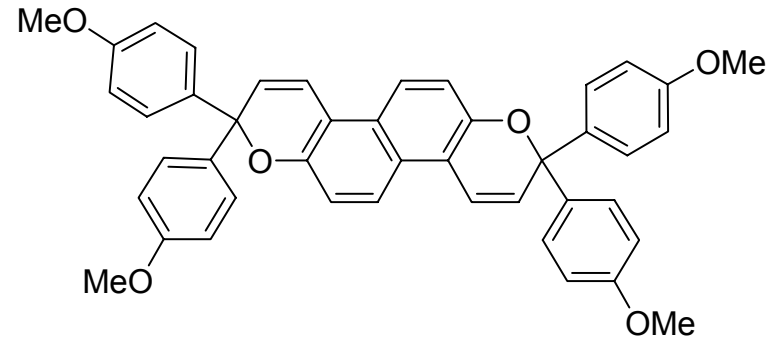

7b. 3,3,9,9-tetra-( $p$-methoxyphenyl)-3H,9H-naphtho[2,1-b:6,5b']dipyran IR (KBr): 3068, 3000, 2860, 1607, 1578, 1509, 1463, 1299, 1252, 1175, 1062, 1033, 838.

${ }^{1} \mathrm{H}-\mathrm{NMR}\left(300 \mathrm{MHz}, \mathrm{CDCl}_{3}\right): \delta 7.77(\mathrm{~d}, \mathrm{~J}=9.0 \mathrm{~Hz}, 2 \mathrm{H}), 7.39-7.32(\mathrm{~m}, 4 \mathrm{H}), 7.19(\mathrm{~d}, \mathrm{~J}=$ $10.0 \mathrm{~Hz}, 2 \mathrm{H}), 7.15(\mathrm{~d}, \mathrm{~J}=9.0 \mathrm{~Hz}, 2 \mathrm{H}), 6.86-6.78(\mathrm{~m}, 4 \mathrm{H}), 6.18(\mathrm{~d}, \mathrm{~J}=10.0 \mathrm{~Hz}, 2 \mathrm{H}), 3.76(\mathrm{~s}$, $12 \mathrm{H})$.

${ }^{13} \mathrm{C}-\mathrm{NMR}\left(75 \mathrm{MHz}, \mathrm{CDCl}_{3}\right): \delta 158.8,148.9,137.1,128.6,128.3,125.4,122.9,119.3,118.8$, 114.7, 113.3, 81.8, 55.2.

HRMS (MALDI) Calcd for $\mathrm{C}_{44} \mathrm{H}_{36} \mathrm{O}_{6}: 660.2506\left(\mathrm{M}^{+}\right)$. Found: 660.2505 (100\%).

Anal. Calcd for $\mathrm{C}_{44} \mathrm{H}_{36} \mathrm{O}_{6}$ : C, 79.98; H, 5.49. Found: C, 80.00; H, 5.61.

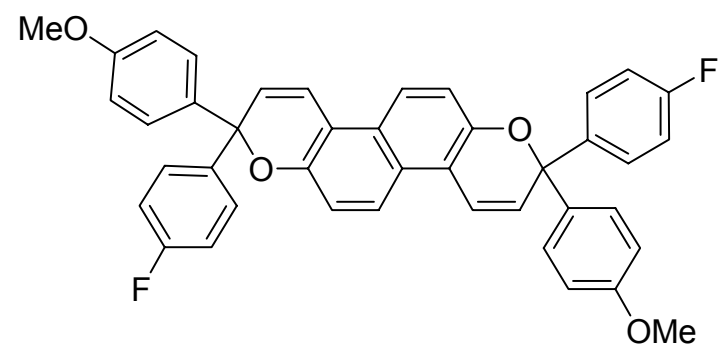

7c. 3,9-di-( $p$-fluorophenyl)-3,9-di-( $p$-methoxyphenyl)-3H,9H-naphtho[2,1-b:6,5b']dipyran IR (KBr): 3056, 2967, 2932, 2838, 1603, 1509, 1299, 1254, 1229, 1178, 1159, 1060, 1030, 835.

${ }^{1} \mathrm{H}-\mathrm{NMR}\left(300 \mathrm{MHz}, \mathrm{CDCl}_{3}\right): \delta 7.78(\mathrm{~d}, \mathrm{~J}=9.1 \mathrm{~Hz}, 2 \mathrm{H}), 7.50-7.28(\mathrm{~m}, 8 \mathrm{H}), 7.21(\mathrm{~d}, \mathrm{~J}=$ $10.3 \mathrm{~Hz}, 2 \mathrm{H}), 7.15(\mathrm{~d}, \mathrm{~J}=9.1 \mathrm{~Hz}, 2 \mathrm{H}), 7.06-6.91(\mathrm{~m}, 4 \mathrm{H}), 6.88-6.76(\mathrm{~m}, 4 \mathrm{H}), 6.17(\mathrm{~d}, \mathrm{~J}=$ $10.0 \mathrm{~Hz}, 2 \mathrm{H}), 3.76(\mathrm{~s}, 6 \mathrm{H})$.

${ }^{13} \mathrm{C}-\mathrm{NMR}\left(75 \mathrm{MHz}, \mathrm{CDCl}_{3}\right): \delta 161.9\left({ }^{1} \mathrm{~J}=246.0 \mathrm{~Hz}\right), 158.8,148.7,140.6,136.5,128.6\left({ }^{3} \mathrm{~J}=\right.$ 7.9Hz), 128.2, 125.4, 123.0, 119.5, 118.7, $114.8\left({ }^{2} \mathrm{~J}=21.4 \mathrm{~Hz}\right), 113.4,81.7,55.3$.

HRMS (MALDI) Calcd. for $\mathrm{C}_{42} \mathrm{H}_{30} \mathrm{~F}_{2} \mathrm{O}_{4}: 636.2106\left(\mathrm{M}^{+}\right)$. Found: 636.2105 (100\%).

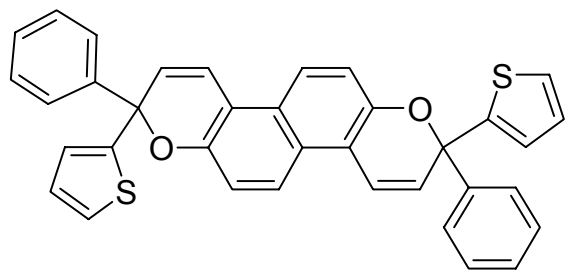

7d. 3,9-di-phenyl-3,9-di-(thiophene-2-yl)-3H,9H-naphtho[2,1-b:6,5b']dipyran IR (KBr): 3088, 1618, 1516, 1490, 1448, 1376, 1252, 1062. 
${ }^{1} \mathrm{H}-\mathrm{NMR}\left(300 \mathrm{MHz}, \mathrm{CDCl}_{3}\right): \delta 7.82(\mathrm{~d}, \mathrm{~J}=9.0 \mathrm{~Hz}, 2 \mathrm{H}), 7.59-7.52(\mathrm{~m}, 4 \mathrm{H}), 7.38-7.24(\mathrm{~m}$, $8 \mathrm{H}), 7.23(\mathrm{~d}, \mathrm{~J}=9.7 \mathrm{~Hz}, 2 \mathrm{H}), 7.21(\mathrm{~d}, \mathrm{~J}=9.0 \mathrm{~Hz}, 2 \mathrm{H}), 6.95-6.87(\mathrm{~m}, 4 \mathrm{H}), 6.27(\mathrm{~d}, \mathrm{~J}=10.0 \mathrm{~Hz}$, $2 \mathrm{H})$.

${ }^{13} \mathrm{C}-\mathrm{NMR}\left(75 \mathrm{MHz}, \mathrm{CDCl}_{3}\right): \delta$ 149.2, 148.8, 144.3, 128.0, 127.8, 127.8, 126.4, 126.3, 126.2, 125.6, 123.1, 119.8, 118.8, 114.6, 80.0.

HRMS (MALDI) Calcd. for $\mathrm{C}_{36} \mathrm{H}_{24} \mathrm{O}_{2} \mathrm{~S}_{2}: 552.1211\left(\mathrm{M}^{+}\right)$. Found: 55.1208 (100\%).

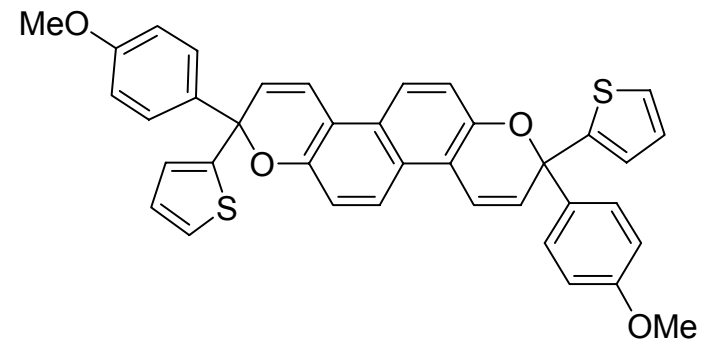

7e. 3,9-di-(p-methoxyphenyl-3,9-di-(thiophene-2-yl)-3H,9H-naphtho[2,1-b:6,5b']dipyran IR (KBr): 3070, 2930, 2834, 1608, 1509, 1461, 1377, 1302, 1251, 1176, 1061, 1031, 830.

${ }^{1} \mathrm{H}-\mathrm{NMR}\left(300 \mathrm{MHz}, \mathrm{CDCl}_{3}\right): \delta 7.80(\mathrm{~d}, \mathrm{~J}=9.1 \mathrm{~Hz}, 2 \mathrm{H}), 7.54-7.41(\mathrm{~m}, 4 \mathrm{H}), 7.32-7.23(\mathrm{~m}, 2 \mathrm{H})$, $7.21(\mathrm{~d}, \mathrm{~J}=10.0 \mathrm{~Hz}, 2 \mathrm{H}), 7.19(\mathrm{~d}, \mathrm{~J}=9.1 \mathrm{~Hz}, 2 \mathrm{H}), 6.96-6.78(\mathrm{~m}, 8 \mathrm{H}), 6.25(\mathrm{~d}, \mathrm{~J}=10.0 \mathrm{~Hz}, 2 \mathrm{H})$, $3.78(\mathrm{~s}, 6 \mathrm{H})$.

${ }^{13} \mathrm{C}-\mathrm{NMR}\left(75 \mathrm{MHz}, \mathrm{CDCl}_{3}\right): \delta 159.1,149.5,148.8,136.4,128.0,127.7,126.2,126.1,125.6$, 123.1, 119.6, 118.8, 114.6, 113.4, 80.0, 55.2.

HRMS (MALDI) Calcd. for $\mathrm{C}_{38} \mathrm{H}_{28} \mathrm{O}_{4} \mathrm{~S}_{2}: 612.1424\left(\mathrm{M}^{+}\right)$. Found: 612.14218 (100\%).

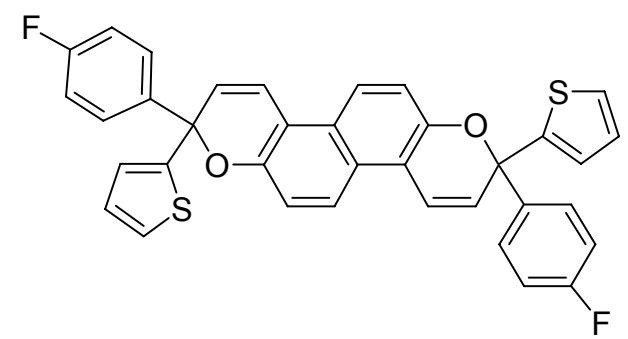

7f. 3,9-di-( $p$-fluorophenyl)-3,9-di-(thiophene-2-yl)-3H,9H-naphtho[2,1-b:6,5b']dipyran IR (KBr): 3072, 1627, 1600, 1519, 1504, 1416, 1379, 1250, 1232, 1221, 1159, 1062, 963, $827,817,750,715$.

${ }^{1} \mathrm{H}-\mathrm{NMR}\left(300 \mathrm{MHz}, \mathrm{CDCl}_{3}\right): \delta 7.82(\mathrm{~d}, \mathrm{~J}=9.0 \mathrm{~Hz}, 2 \mathrm{H}), 7.58-7.46(\mathrm{~m}, 4 \mathrm{H}), 7.30-7.25(\mathrm{~m}, 2 \mathrm{H})$, $7.23(\mathrm{~d}, \mathrm{~J}=10.0 \mathrm{~Hz}, 2 \mathrm{H}), 7.19(\mathrm{~d}, \mathrm{~J}=9.0 \mathrm{~Hz}, 2 \mathrm{H}), 7.07-6.95(\mathrm{~m}, 4 \mathrm{H}), 6.94-6.86(\mathrm{~m}, 4 \mathrm{H}), 6.24$ $(\mathrm{d}, \mathrm{J}=10.0 \mathrm{~Hz}, 2 \mathrm{H})$.

${ }^{13} \mathrm{C}-\mathrm{NMR}\left(75 \mathrm{MHz}, \mathrm{CDCl}_{3}\right): \delta 162.1\left({ }^{1} \mathrm{~J}=245.6 \mathrm{~Hz}\right), 148.8,148.6,140.0,128.1\left({ }^{3} \mathrm{~J}=8.6 \mathrm{~Hz}\right)$, $127.5,126.3,125.5,123.1,119.9,118.8,114.9\left({ }^{2} \mathrm{~J}=22.0 \mathrm{~Hz}\right), 114.5,79.8$.

HRMS (MALDI) Calcd. for $\mathrm{C}_{36} \mathrm{H}_{22} \mathrm{~F}_{2} \mathrm{O}_{2} \mathrm{~S}_{2}: 589.1102\left(\mathrm{MH}^{+}\right)$. Found: 598.1097 (58\%).

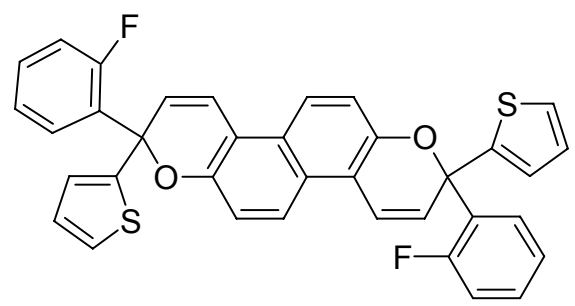

7g. 3,9-di-(o-fluorophenyl)-3,9-di-(thiophene-2-yl)-3H,9H-naphtho[2,1-b:6,5b']dipyran IR (KBr): 3070, 1626, 1583, 1518, 1483, 1454, 1379, 1322, 1272, 1238, 1200, 1106, 1063, $960,897,838,754,710$. 
${ }^{1} \mathrm{H}-\mathrm{NMR}\left(300 \mathrm{MHz}, \mathrm{CDCl}_{3}\right): \delta 7.84(\mathrm{~d}, \mathrm{~J}=9.3 \mathrm{~Hz}, 2 \mathrm{H}), 7.85-7.70(\mathrm{~m}, 2 \mathrm{H}), 7.33-7.19(\mathrm{~m}, 8 \mathrm{H})$, 7.18-7.08 (m, 2H), 7.08-6.98 (m, 2H), 6.98-6.86 (m, 4H), $6.50(\mathrm{dd}, \mathrm{J}=10.0,5.0 \mathrm{~Hz}, 2 \mathrm{H})$.

${ }^{13} \mathrm{C}-\mathrm{NMR}\left(75 \mathrm{MHz}, \mathrm{CDCl}_{3}\right): \delta 159.2\left({ }^{1} \mathrm{~J}=247.2 \mathrm{~Hz}\right), 148.4,147.8,130.9\left({ }^{2} \mathrm{~J}=11.0 \mathrm{~Hz}\right), 129.7$ $\left({ }^{3} \mathrm{~J}=8.6 \mathrm{~Hz}\right), 127.4,126.3,126.0,125.6,123.7,123.1,119.7,118.6,116.3\left({ }^{2} \mathrm{~J}=22.0 \mathrm{~Hz}\right)$, 114.4, 78.0.

HRMS (MALDI) Calcd. for $\mathrm{C}_{36} \mathrm{H}_{22} \mathrm{~F}_{2} \mathrm{O}_{2} \mathrm{~S}_{2}: 611.0922$ (MNa ${ }^{+}$). Found: 611.0917 (87\%).

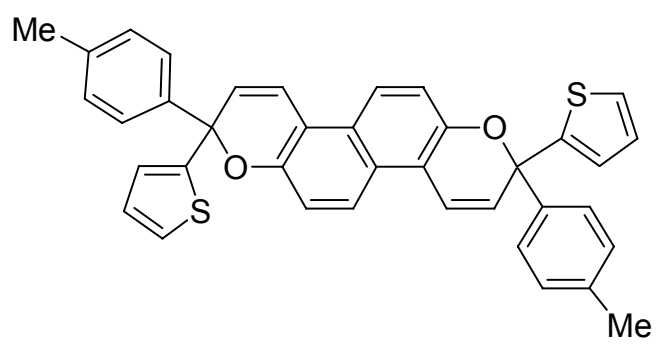

7h. 3,9-di-( $p$-methylphenyl)-3,9-di-(thiophene-2-yl)-3H,9H-naphtho[2,1-b:6,5b']dipyran IR (KBr): 3088, 3022, 2918, 1625, 1519, 1510, 1382, 1254, 1236, 1219, 1185, 1071, 959, 816, 804, 704.

${ }^{1} \mathrm{H}-\mathrm{NMR}\left(300 \mathrm{MHz}, \mathrm{CDCl}_{3}\right): \delta 7.80(\mathrm{~d}, \mathrm{~J}=9.0 \mathrm{~Hz}, 2 \mathrm{H}), 7.44(\mathrm{~d}, \mathrm{~J}=7.8 \mathrm{~Hz}, 4 \mathrm{H}), 7.30-7.24(\mathrm{~m}$, $2 \mathrm{H}), 7.21(\mathrm{~d}, \mathrm{~J}=10.0 \mathrm{~Hz}, 2 \mathrm{H}), 7.19(\mathrm{~d}, \mathrm{~J}=9.0 \mathrm{~Hz}, 2 \mathrm{H}), 7.14(\mathrm{dd}, \mathrm{J}=7.8,0.6 \mathrm{~Hz}, 4 \mathrm{H}), 6.95-$ $6.85(\mathrm{~m}, 4 \mathrm{H}), 6.27(\mathrm{~d}, \mathrm{~J}=10.0 \mathrm{~Hz}, 2 \mathrm{H}), 2.32(\mathrm{~s}, 6 \mathrm{H})$.

${ }^{13} \mathrm{C}-\mathrm{NMR}\left(75 \mathrm{MHz}, \mathrm{CDCl}_{3}\right): \delta 149.3,148.7,141.3,137.4,128.7,127.9,126.2,125.5,123.0$, 119.6, 118.8, 114.5, 80.0, 21.2.

HRMS (MALDI) Calcd. for $\mathrm{C}_{38} \mathrm{H}_{28} \mathrm{O}_{2} \mathrm{~S}_{2}: 603.1243\left(\mathrm{MNa}^{+}\right)$. Found: 603.1429 (51\%).

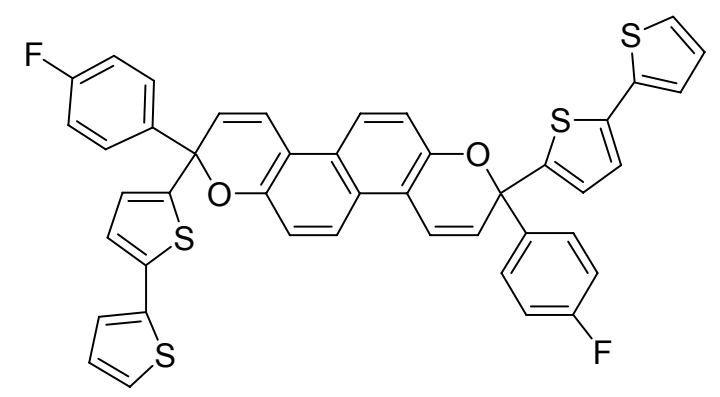

7i. 3,9-di-(2,2'-bithiophene-5-yl)-3,9-di-( $p$-fluorophenyl)-3H,9H-naphtho[2,1-b:6,5b']dipyran IR (KBr): 3069, 1626, 1601, 1506, 1415, 1379, 1324, 1228, 1158, 1062, 1014, 954, 837, 800, $745,695$.

${ }^{1} \mathrm{H}-\mathrm{NMR}\left(300 \mathrm{MHz}, \mathrm{CDCl}_{3}\right): \delta 7.84(\mathrm{~d}, \mathrm{~J}=9.0 \mathrm{~Hz}, 2 \mathrm{H}), 7.62-7.52(\mathrm{~m}, 4 \mathrm{H}), 7.25(\mathrm{~d}, \mathrm{~J}=$ $10.0 \mathrm{~Hz}, 2 \mathrm{H}), 7.22(\mathrm{~d}, \mathrm{~J}=9.0 \mathrm{~Hz}, 2 \mathrm{H}), 7.17(\mathrm{~d}, \mathrm{~J}=4.0 \mathrm{~Hz}, 2 \mathrm{H}), 7.09(\mathrm{dd}, \mathrm{J}=3.7,1.3 \mathrm{~Hz}, 2 \mathrm{H})$, 7.08-6.99 (m, 4H), $6.97(\mathrm{~d}, \mathrm{~J}=4.0 \mathrm{~Hz}, 2 \mathrm{H}), 6.95(\mathrm{~d}, \mathrm{~J}=3.7 \mathrm{~Hz}, 2 \mathrm{H}), 6.82(\mathrm{~d}, \mathrm{~J}=3.7 \mathrm{~Hz}, 2 \mathrm{H})$, $6.22(\mathrm{~d}, \mathrm{~J}=10.0 \mathrm{~Hz}, 2 \mathrm{H})$.

${ }^{13} \mathrm{C}-\mathrm{NMR}\left(75 \mathrm{MHz}, \mathrm{CDCl}_{3}\right): \delta 162.1\left({ }^{1} \mathrm{~J}=246.6 \mathrm{~Hz}\right), 148.5,147.5,139.7,138.4,136.9,128.1$ $\left({ }^{3} \mathrm{~J}=8.5 \mathrm{~Hz}\right), 127.6,127.2,127.1,127.1,125.7,124.5,123.7,123.3,122.7,120.1,118.8$, $115.0\left({ }^{2} \mathrm{~J}=21.4 \mathrm{~Hz}\right), 114.5,79.8$.

HRMS (MALDI) Calcd. for $\mathrm{C}_{44} \mathrm{H}_{26} \mathrm{~F}_{2} \mathrm{O}_{2} \mathrm{~S}_{4}: 753.0857\left(\mathrm{MH}^{+}\right)$. Found: 753.0851 (100\%). 


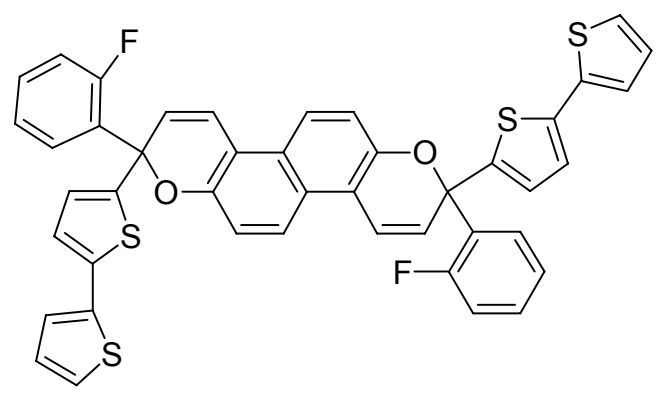

7j. 3,9-di-(2,2'-bithiophene-5-yl)-3,9-di-(o-fluorophenyl)-3H,9H-naphtho[2,1-b:6,5b']dipyran IR (KBr): 3070, 1629, 1610, 1580, 1518, 1482, 1452, 1416, 1380, 1324, 1271, 1242, 1203, 1105, 1063, 954, 904, 839, 821, 799, 752, 697.

${ }^{1} \mathrm{H}-\mathrm{NMR}\left(300 \mathrm{MHz}, \mathrm{CDCl}_{3}\right): \delta 7.86(\mathrm{~d}, \mathrm{~J}=9.0 \mathrm{~Hz}, 2 \mathrm{H}), 7.82-7.73(\mathrm{~m}, 2 \mathrm{H}), 7.35-7.26(\mathrm{~m}, 2 \mathrm{H})$, $7.27(\mathrm{~d}, \mathrm{~J}=9.0 \mathrm{~Hz}, 2 \mathrm{H}), 7.25(\mathrm{~d}, \mathrm{~J}=9.7 \mathrm{~Hz}, 2 \mathrm{H}), 7.20-7.10(\mathrm{~m}, 4 \mathrm{H}), 7.10-7.04(\mathrm{~m}, 3 \mathrm{H}), 7.03$ $(\mathrm{dd}, \mathrm{J}=8.1 \mathrm{~Hz}, 0.9 \mathrm{~Hz}, 1 \mathrm{H}), 6.99-6.90(\mathrm{~m}, 4 \mathrm{H}), 6.86(\mathrm{~d}, \mathrm{~J}=4.0 \mathrm{~Hz}, 2 \mathrm{H}), 6.48(\mathrm{dd}, \mathrm{J}=$ $10.0,4.7 \mathrm{~Hz}, 2 \mathrm{H})$.

${ }^{13} \mathrm{C}-\mathrm{NMR}\left(75 \mathrm{MHz}, \mathrm{CDCl}_{3}\right): \delta 159.1\left({ }^{1} \mathrm{~J}=248.4 \mathrm{~Hz}\right), 148.4,146.4,138.2,137.1,130.5\left({ }^{2} \mathrm{~J}=\right.$ $19.0 \mathrm{~Hz}), 129.8\left({ }^{3} \mathrm{~J}=7.9 \mathrm{~Hz}\right), 127.6,127.4,126.7,125.8\left({ }^{3} \mathrm{~J}=4.3 \mathrm{~Hz}\right), 125.6,124.4,123.7$, 123.2, 122.9, 120.0, 118.7, $116.4\left({ }^{2} \mathrm{~J}=22.0 \mathrm{~Hz}\right), 114.5,78.0$.

HRMS (MALDI) Calcd. for $\mathrm{C}_{44} \mathrm{H}_{26} \mathrm{~F}_{2} \mathrm{O}_{2} \mathrm{~S}_{4}: 753.0857\left(\mathrm{MH}^{+}\right)$. Found: 753.0856 (100\%).

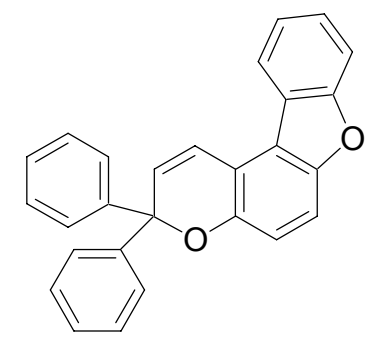

8. 3,3-diphenyl-[3H]-benzofuro[3,2-f]chromene.

IR (KBr): 3056, 3032, 1487, 1472, 1446, 1427, 1244, 1187, 1148, 1092, 988, 745, 698.

${ }^{1} \mathrm{H}-\mathrm{NMR}\left(300 \mathrm{MHz}, \mathrm{CDCl}_{3}\right): \delta 8.10(\mathrm{dd}, \mathrm{J}=7.8,0.6 \mathrm{~Hz}, 1 \mathrm{H}), 7.57-7.40(\mathrm{~m}, 6 \mathrm{H}), 7.38(\mathrm{~d}, \mathrm{~J}=$ $10.0 \mathrm{~Hz}, 1 \mathrm{H}), 7.37-7.20(\mathrm{~m}, 8 \mathrm{H}), 7.06(\mathrm{~d}, \mathrm{~J}=8.7 \mathrm{~Hz}, 1 \mathrm{H}), 6.40(\mathrm{~d}, \mathrm{~J}=9.7 \mathrm{~Hz}, 1 \mathrm{H})$.

${ }^{13} \mathrm{C}-\mathrm{NMR}\left(75 \mathrm{MHz}, \mathrm{CDCl}_{3}\right): \delta 157.1,151.4,148.2,144.7,130.6,128.2,127.7,127.2,127.0$, 124.3, 122.6, 122.2, 120.6, 116.3, 115.7, 111.8, 111.8, 111.7, 82.2.

MS: 374.2( $\left.\mathrm{M}^{+}\right)$.

Anal. Calcd for $\mathrm{C}_{27} \mathrm{H}_{18} \mathrm{O}_{2}$ : C, 86.61; H, 4.85. Found: C, 86.67; H, 5.05.

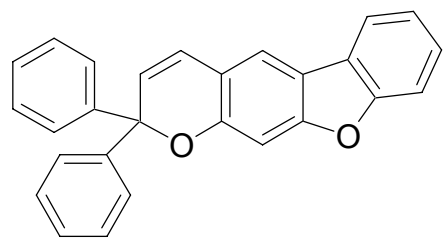

9. 7,7-diphenyl-[7H]-benzofuro[2,3-g]chromene.

IR (KBr): 3025, 1631, 1487, 1459, 1436, 1318, 1241, 1220, 1196, 1165, 992, 944, 870, 700.

${ }^{1} \mathrm{H}-\mathrm{NMR}\left(300 \mathrm{MHz}, \mathrm{CDCl}_{3}\right): \delta 7.84(\mathrm{~d}, \mathrm{~J}=9.0 \mathrm{~Hz}, 1 \mathrm{H}), 7.54-7.45(\mathrm{~m}, 6 \mathrm{H}), 7.40(\mathrm{ddd}, \mathrm{J}=8.4$, $7.2,1.2 \mathrm{~Hz}, 1 \mathrm{H}), 7.37-7.22(\mathrm{~m}, 7 \mathrm{H}), 7.20(\mathrm{~s}, 1 \mathrm{H}), 6.76(\mathrm{~d}, \mathrm{~J}=9.9 \mathrm{~Hz}, 1 \mathrm{H}), 6.30(\mathrm{~d}, 1 \mathrm{H}, \mathrm{J}=$ 9.7 Hz).

${ }^{13} \mathrm{C}-\mathrm{NMR}\left(75 \mathrm{MHz}, \mathrm{CDCl}_{3}\right): \delta 157.1,148.7,144.8,130.4,128.3,127.6,127.2,124.4,124.1$, 122.6, 121.2, 120.7, 111.6, 111.0, 108.9, 107.9, 82.7. 
MS: $374.2\left(\mathrm{M}^{+}\right)$.

Anal. Calcd for $\mathrm{C}_{27} \mathrm{H}_{18} \mathrm{O}_{2}$ : C, 86.61; $\mathrm{H}, 4$.85. Found: C, 86.47; H, 5.08.

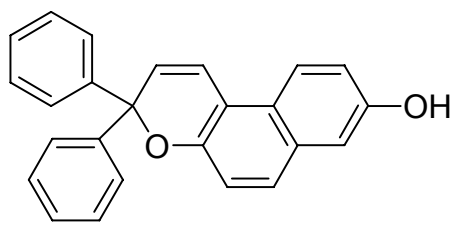

10. 3,3-diphenyl-8-hydroxy-[3H][2,1-b]naphthopyran.

IR (KBr): 3616, 3059, 1618, 1593, 1518, 1490, 1446, 1380, 1250, 1220, 1189, 1083, 1009, 860, 749, 728, 696.

${ }^{1} \mathrm{H}-\mathrm{NMR}\left(300 \mathrm{MHz}, \mathrm{CDCl}_{3}\right): \delta 7.86(\mathrm{~d}, \mathrm{~J}=9.0 \mathrm{~Hz}, 1 \mathrm{H}), 7.52-7.45(\mathrm{~m}, 5 \mathrm{H}), 7.36-7.20(\mathrm{~m}, 7 \mathrm{H})$, $7.17(\mathrm{~d}, \mathrm{~J}=8.7 \mathrm{~Hz}, 1 \mathrm{H}), 7.09(\mathrm{dd}, \mathrm{J}=9.0,2.5 \mathrm{~Hz}, 1 \mathrm{H}), 7.04(\mathrm{~d}, \mathrm{~J}=2.5 \mathrm{~Hz}, 1 \mathrm{H}), 6.27(\mathrm{~d}, \mathrm{~J}=$ $10.0 \mathrm{~Hz}, 1 \mathrm{H}), 4.75(\mathrm{~s}, 1 \mathrm{H})$.

${ }^{13} \mathrm{C}-\mathrm{NMR}\left(\mathrm{CDCl}_{3}\right): \delta 151.6,148.9,144.8,130.3,128.0,128.0,127.0,125.0,123.2,119.5$, 119.0, 118.1, 114.2, 110.3, 82.3.

MS: $350.2\left(\mathrm{M}^{+}\right)$.

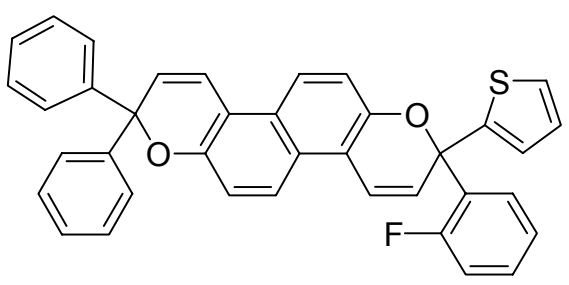

11. 3,3-di-phenyl-9-(o-fluorophenyl)-9-(thiophene-2-yl)-3H,9H-naphtho[2,1-b:6,5b']dipyran IR (KBr): 3059, 1627, 1517, 1483, 1448, 1379, 1322, 1240, 1222, 1193, 1166, 1060, 959, $838,822,750,701$.

${ }^{1} \mathrm{H}-\mathrm{NMR}\left(300 \mathrm{MHz}, \mathrm{CD}_{2} \mathrm{Cl}_{2}\right): \delta 7.88(\mathrm{~d}, \mathrm{~J}=7.8 \mathrm{~Hz}, 1 \mathrm{H}), 7.83(\mathrm{~d}, \mathrm{~J}=7.8 \mathrm{~Hz}, 1 \mathrm{H}), 7.75(\mathrm{~d} t, \mathrm{~J}=$ 7.8, $1.6 \mathrm{~Hz}, 1 \mathrm{H}), 7.51-7.43(\mathrm{~m}, 4 \mathrm{H}), 7.36-7.19(\mathrm{~m}, 12 \mathrm{H}), 7.14(\mathrm{dt}, \mathrm{J}=7.8,1.2 \mathrm{~Hz}, 1 \mathrm{H}), 7.02$ $(\mathrm{ddd}, \mathrm{J}=11.8,8.1,1.2 \mathrm{~Hz}, 1 \mathrm{H}), 6.95(\mathrm{dd}, \mathrm{J}=3.7,0.9 \mathrm{~Hz}, 1 \mathrm{H}), 6.91(\mathrm{dd}, \mathrm{J}=5.0,3.7 \mathrm{~Hz}, 1 \mathrm{H})$, $6.48(\mathrm{~d}, \mathrm{~J}=10.0,5.0 \mathrm{~Hz}, 1 \mathrm{H}), 6.34(\mathrm{~d}, \mathrm{~J}=10.0 \mathrm{~Hz}, 1 \mathrm{H})$.

${ }^{13} \mathrm{C}-\mathrm{NMR}\left(75 \mathrm{MHz}, \mathrm{CDCl}_{3}\right): \delta 159.2\left({ }^{1} \mathrm{~J}=248.4 \mathrm{~Hz}\right), 149.0,148.2,147.8,144.6,130.9\left({ }^{2} \mathrm{~J}=\right.$ $11.0 \mathrm{~Hz}), 129.7\left({ }^{3} \mathrm{~J}=7.9 \mathrm{~Hz}\right), 128.2,128.0,127.4,126.9,126.8,126.3,126.2,126.1,126.0$, $125.6,125.3,123.7,123.1,122.9,119.7,119.4,118.9,118.5,116.4,116.2,114.5\left({ }^{2} \mathrm{~J}=\right.$ $18.3 \mathrm{~Hz}), 82.3,77.9$.

HRMS (MALDI) Calcd. for $\mathrm{C}_{38} \mathrm{H}_{25} \mathrm{FO}_{2} \mathrm{~S}: 564.1554\left(\mathrm{M}^{+}\right)$. Found: 564.1560 (94\%).

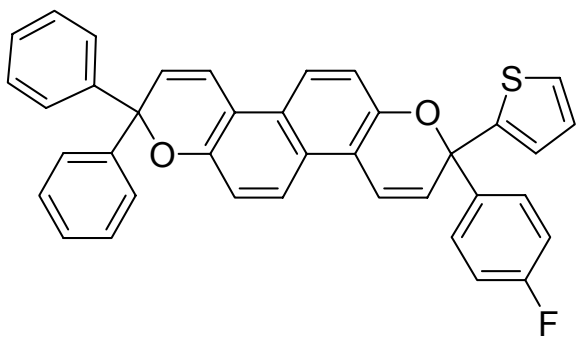

12. 3,3-di-phenyl-9-(p-fluorophenyl)-9-(thiophene-2-yl)-3H,9H-naphtho[2,1-b:6,5b']dipyran IR (KBr): 3057, 1622, 1601, 1519, 1506, 1492, 1446, 1378, 1324, 1224, 1187, 1157, 1065 , 969, 828, 810, 750, 698.

${ }^{1} \mathrm{H}-\mathrm{NMR}\left(300 \mathrm{MHz}, \mathrm{CDCl}_{3}\right): \delta 7.81(\mathrm{~d}, \mathrm{~J}=9.0 \mathrm{~Hz}, 1 \mathrm{H}), 7.79(\mathrm{~d}, \mathrm{~J}=9.0 \mathrm{~Hz}, 1 \mathrm{H}), 7.56-7.48(\mathrm{~m}$, $2 \mathrm{H}), 7.48-7.42(\mathrm{~m}, 4 \mathrm{H}), 7.34-7.29(\mathrm{~m}, 3 \mathrm{H}), 7.39-7.19(\mathrm{~m}, 7 \mathrm{H}), 7.18(\mathrm{~d}, \mathrm{~J}=9.7 \mathrm{~Hz}, 1 \mathrm{H}), 7.05-$ $6.95(\mathrm{~m}, 2 \mathrm{H}), 6.92-6.87(\mathrm{~m}, 2 \mathrm{H}), 6.26(\mathrm{~d}, \mathrm{~J}=10.0 \mathrm{~Hz}, 1 \mathrm{H}), 6.23(\mathrm{~d}, \mathrm{~J}=9.7 \mathrm{~Hz}, 1 \mathrm{H})$. 
${ }^{13} \mathrm{C}-\mathrm{NMR}\left(75 \mathrm{MHz}, \mathrm{CDCl}_{3}\right): \delta 162.0\left({ }^{1} \mathrm{~J}=246.0 \mathrm{~Hz}\right), 149.0,148.9,148.4,144.6,140.0,128.3$, $128.1\left({ }^{3} \mathrm{~J}=7.9 \mathrm{~Hz}\right), 128.0,127.9,127.4,126.8,126.3,125.6,125.3,123.2,122.9,119.9$, 119.4, 118.9, 118.6, $114.8\left({ }^{2} \mathrm{~J}=22.0 \mathrm{~Hz}\right), 114.4,82.3$, 79.7.

HRMS (MALDI) Calcd. for $\mathrm{C}_{38} \mathrm{H}_{25} \mathrm{FO}_{2} \mathrm{~S}: 564.1554$ (M ${ }^{+}$). Found: 564.1561 (87\%). 